\title{
Clinical Outcomes of Definitive and Postoperative Radiotherapy for Stage I-IVB Hypopharyngeal Cancer
}

\author{
KATSUYUKI SHIRAI ${ }^{1}$, JUN-ICHI SAITOH ${ }^{1}$, ATSUSHI MUSHA ${ }^{1}$, TAKANORI ABE ${ }^{1}$, DAIJIRO KOBAYASHI ${ }^{1}$, \\ YOSUKE TAKAKUSAGI ${ }^{1}$, YUKIHIRO TAKAYSU ${ }^{2}$, MASATO SHINO $^{2}$, MINORU TOYODA $^{2}$, \\ KATSUMASA TAKAHASHI ${ }^{2}$, KAZUAKI CHIKAMATSU ${ }^{2}$, TATSUYA OHNO ${ }^{1}$ and TAKASHI NAKANO ${ }^{1}$ \\ ${ }^{1}$ Department of Radiation Oncology, Gunma University Graduate School of Medicine, Maebashi, Japan; \\ ${ }^{2}$ Department of Otolaryngology-Head and Neck Surgery, \\ Gunma University Graduate School of Medicine, Maebashi, Japan
}

\begin{abstract}
Background: Hypopharyngeal cancer is relatively rare disease and continues to have a poor prognosis. This study analyzed the efficacy and safety of radiotherapy for stage I-IVB hypopharyngeal cancer. Patients and Methods: Between 2000 and 2015, 72 patients were treated with definitive radiotherapy and 29 patients with stage IVA were treated with postoperative radiotherapy. Results: With definitive radiotherapy, the 3-year locoregional control rates for stage I-II, III, IVA, and IVB disease were $89 \%, 74 \%, 51 \%$ and $0 \%$, respectively. The 3-year overall survival rates for patients with stage I-II, III, IVA and IVB disease were $84 \%$, $89 \%, 55 \%$ and $15 \%$, respectively. In patients with stage IVA disease treated with postoperative radiotherapy, 3-year locoregional control and overall survival rates were $83 \%$ and $75 \%$, respectively, which were significantly better than those treated with definitive radiotherapy. Conclusion: Definitive radiotherapy was effective for stage I-III disease. Surgery and postoperative radiotherapy improved the survival rate of patients with stage IVA hypopharyngeal cancer.
\end{abstract}

Most patients with hypopharyngeal cancer are initially diagnosed as having advanced-stage disease with neck swelling or dysphagia, this disease continues to have a poor prognosis. Surgery has been the mainstay of curative treatment, but a major problem is that it reduces quality of life, such as loss of voice. In order to achieve the best outcomes to

This article is freely accessible online.

Correspondence to: Katsuyuki Shirai, MD, Ph.D., Department of Radiation Oncology, Gunma University Graduate School of Medicine, Maebashi, Gunma 371-8511 Japan. Tel: +81 272208383, Fax: +81272208397, e-mail: kshirai@gunma-u.ac.jp

Key Words: Hypopharyngeal cancer, definitive radiotherapy, postoperative radiotherapy, chemoradiotherapy, induction chemotherapy. improve organ function, multimodal treatment is important (1). Although many clinical trials include cancer types with multiple sites in the head and neck, few studies have analyzed only hypopharyngeal cancer, owing to the rarity of this disease. Moreover, because cancer of the head and neck can have diverse characteristics and distinct outcomes, it is important to analyze the primary tumor sites separately.

Radiotherapy is a treatment option achieving complete response with laryngeal preservation. However, the use of radiotherapy alone has been reported to have poor local control for hypopharyngeal cancer (2). Several studies of unresectable advanced hypopharyngeal cancer reported that chemoradiotherapy improved overall survival $(3,4)$. Recently, a meta-analysis showed that concurrent chemoradiotherapy improved local control and overall survival rates compared to radiotherapy alone in advanced head and neck cancer (5). Based on these encouraging results, chemoradiotherapy has been considered the definitive treatment for advanced head and neck cancer, including hypopharyngeal cancer. However, hypopharyngeal cancer usually comprised a smaller subgroup (5-39\%) in most studies, and details of clinical results were seldom specifically reported.

Therefore, we retrospectively analyzed the efficacy and safety of definitive radiotherapy for stage I-IVB hypopharyngeal cancer treated at our hospital.

\section{Patients and Methods}

This retrospective study was approved by the Institutional Review Board (number: 15-37). Between January 2000 and June 2015, 101 patients with hypopharyngeal cancer were consecutively irradiated at Gunma University Hospital. Patients treated with radiotherapy alone, concurrent chemoradiotherapy, and induction chemotherapy were included. Patients treated with preoperative radiotherapy and palliative radiotherapy were excluded. There were 72 patients (stage I/II/III/IVA/IVB: 4/17/12/27/12) treated with definitive radiotherapy and 29 patients with stage IVA disease treated with postoperative radiotherapy. Their clinical characteristics are summarized in Table I. 
The median age was 66 years (range $=44-92$ years), and the number of male patients was 94 (93\%). All patients were pathologically diagnosed by biopsy. Most patients (99\%) were diagnosed as having squamous cell carcinoma, with basaloid squamous cell carcinoma in one and lymphoepithelial carcinoma in one. The primary tumor subsites were the pyriform fossa in 87 patients $(86 \%)$, the posterior wall in eight $(8 \%)$, and the postcricoid region in six $(6 \%)$. The pretreatment evaluations included a physical examination, laryngoscopy, computed tomography (CT), and magnetic resonance imaging (MRI). Since 2005, ${ }^{18}$ F-fluorodeoxyglucose-positron-emission tomography (FDG-PET) has also been performed.

At the time of hypopharyngeal cancer diagnosis, 14 patients (14\%) had no symptoms. Cancer was coincidentally found for all asymptomatic patients when they underwent gastrointestinal endoscopy for other diseases, and most (93\%) were diagnosed with stage I-II hypopharyngeal cancer. Among 87 symptomatic patients $(86 \%)$, there were 25 with neck swelling, 21 with discomfort in their pharynx, 20 with pain in their pharynx, eight with dysphagia, eight with hoarseness, three with dyspnea, one with appetite loss, and one with hemosputum. Among 25 patients with neck swelling, definitive radiotherapy $(n=22,88 \%)$ was performed significantly more often than surgery followed by postoperative radiotherapy $(\mathrm{n}=3,12 \%)(p=0.01)$.

All patients provided informed consent before treatments. The median radiation doses for definitive radiotherapy and postoperative radiotherapy were $66 \mathrm{~Gy}$ (range $=50-72 \mathrm{~Gy}$ ) and $60 \mathrm{~Gy}$ (range $=52$ 64 Gy), respectively. Three-dimensional conformal radiotherapy used parallel-opposed lateral fields with elective nodal irradiation. After administration of $40 \mathrm{~Gy}$ for elective nodal irradiation, a shrinking field was delivered to avoid the spinal cord. Intensitymodulated radiotherapy was performed in 19 patients (19\%).

Concurrent chemoradiotherapy was performed in 86 patients $(87 \%)$, and a low-dose cisplatin and docetaxel regimen was used in 62 patients; cisplatin was delivered at $6 \mathrm{mg} / \mathrm{m}^{2}$ continuously in 20 fractions for 4 weeks, and docetaxel was delivered weekly at $10 \mathrm{mg} / \mathrm{m}^{2}$ in four fractions (Figure 1). Details of this regimen have been described previously (6). Weekly docetaxel alone without cisplatin was used in 10 patients, and low-dose cisplatin alone without docetaxel was used in two because of inadequate renal or liver function or poor general condition. High-dose cisplatin was used in eight patients, cetuximab was used in four patients, and nedaplatin plus 5-fluorouracil was used in one patient.

Induction chemotherapy was performed in 38 patients $(38 \%)$, especially for locally advanced hypopharyngeal cancer. TPF therapy $\left(60 \mathrm{mg} / \mathrm{m}^{2}\right.$ docetaxel, $60 \mathrm{mg} / \mathrm{m}^{2}$ cisplatin and $700 \mathrm{mg} / \mathrm{m}^{2} 5-$ fluorouracil), administered as a continuous 24-hour infusion for 4 days, was used in 22 patients. FP therapy $\left(80 \mathrm{mg} / \mathrm{m}^{2}\right.$ cisplatin and $800 \mathrm{mg} / \mathrm{m}^{2} 5$-fluorouracil), administered as a continuous 24 -hour infusion for 5 days, was used in 16 patients.

After the end of treatment, patients were seen every month for the first year and every 3 months thereafter. Follow-up consisted of physical examination, laryngoscopy, CT, MRI, and FDG-PET. Common Terminology Criteria for Adverse Events version 4.0 was used to evaluate acute and late adverse events (7).

Locoregional control, progression-free survival, and overall survival rates were statistically calculated using the Kaplan-Meier method and compared using log-rank tests. Ultimate laryngeal preservation was defined when all the following requirements were met: (i) no evidence of local failure, (ii) no need for feeding tube or tracheostomy, and (iii) no subsequent total laryngectomy, excluding partial resection. Multivariate analysis was performed with a Cox regression hazard model. Differences between groups were calculated using the $t$-test. A $p$-value of less than 0.05 was defined as acceptable statistical significance.

\section{Results}

The median follow-up time for surviving patients was 35 months (range=10.7-118.7 months). Initial failure sites are shown in Table II. Considering all 101 patients, 29 (29\%) had locoregional recurrences (10 with local recurrences, 12 with lymph node recurrences, and 7 with local and lymph node recurrences). Distant metastases were observed in 10 patients (lung, 4; mediastinum, 1; bone, 1; pleura, 1; liver, 1 ; and multiple sites, 3 ).

In the definitive radiotherapy group, 3-year locoregional control rates were $89 \%$ in stage I-II, $74 \%$ in stage III, $51 \%$ in stage IVA, and $0 \%$ in stage IVB cases (Figure 2A). Stage was a significant negative prognostic factor for locoregional control $(p<0.01)$. The subsite of primary tumor and concurrent chemoradiotherapy were not significantly associated with locoregional control. For stage III and IV disease, the induction chemotherapy group had a significantly worse 3-year locoregional control rate than did the non-induction group $(37 \%$ vs. $58 \%$, respectively, $p=0.04)$. Salvage surgery was performed in four patients with local recurrences of the hypopharynx (three with total laryngopharyngectomy and one with partial resection for laryngeal preservation) and two patients with cervical lymph node metastasis (radical neck resection). There were no severe postoperative complications.

Ultimate laryngeal preservation rates were $90 \%(n=19 / 21)$ in stage I-II, $83 \%(n=10 / 12)$ in stage III, $77 \%(n=21 / 27)$ in stage IVA, and $50 \%(n=6 / 12)$ in stage IVB.

Three-year progression-free survival rates were $75 \%$ in those with stage I-II, 74\% in those with stage III, 36\% in those with stage IVA, and $0 \%$ in those with stage IVB disease (Figure 2B). Stage was a significant negative prognostic factor for progression-free survival $(p<0.01)$.

During follow-up of the definitive radiotherapy group, 20 patients died of hypopharyngeal cancer, and seven died of intercurrent disease, including two of esophageal cancer, one of tongue cancer, one of lung cancer, one of pneumonia, one of chronic heart failure, and one of acute subdural hematoma. The 3-year overall survival rates for patients with stage I-II, III, IVA and IVB in this group were $84 \%, 89 \%, 55 \%$ and $15 \%$, respectively (Figure $2 \mathrm{C}$ ). Stage was a significant negative prognostic factor for overall survival $(p<0.01)$. Asymptomatic patients tended to have a better 3-year overall survival rate than did symptomatic patients (92\% vs. 64\%, $p=0.08)$. Patients with neck swelling had a significantly worse survival rate than did other patients $(76 \%$ vs. $46 \%, p<0.01)$. There were no significant associations between overall survival rate 
Table I. Characteristics of 101 patients treated with definitive and postoperative radiotherapy.

\begin{tabular}{lcc}
\hline Characteristic & $\begin{array}{c}\text { Definitive } \\
\text { radiotherapy } \\
(\mathrm{n}=72)\end{array}$ & $\begin{array}{c}\text { Postoperative } \\
\text { radiotherapy } \\
(\mathrm{n}=29)\end{array}$ \\
\hline Median age (range), years & $67(44-92)$ & $62(45-81)$ \\
Gender, n (\%) & $67(93 \%)$ & $27(93 \%)$ \\
Male & $5(7 \%)$ & $2(7 \%)$ \\
Female & & \\
Performance status, n (\%) & $69(96 \%)$ & $29(100 \%)$ \\
0/1 & $3(4 \%)$ & $0(0 \%)$ \\
2 & $4(6 \%)$ & $0(0 \%)$ \\
Stage, n (\%) & $17(24 \%)$ & $0(0 \%)$ \\
I & $12(17 \%)$ & $0(0 \%)$ \\
II & $27(38 \%)$ & $29(100 \%)$ \\
III & $12(17 \%)$ & $0(0 \%)$ \\
IVA & & \\
IVB & $61(85 \%)$ & $26(90 \%)$ \\
Subsite, n (\%) & $7(10 \%)$ & $1(3 \%)$ \\
Pyriform fossa & $4(6 \%)$ & $2(7 \%)$ \\
Posterior wall & & \\
Postcricoid region & $71(99 \%)$ & $28(97 \%)$ \\
Histological subtype & $1(1 \%)$ & $1(3 \%)$ \\
Squamous cell carcinoma & $66(50-72)$ & $60(52-64)$ \\
Other & $65(90 \%)$ & $22(76 \%)$ \\
Median radiation dose (range), Gy & $7(10 \%)$ & $7(24 \%)$ \\
Concurrent chemoradiotherapy, n (\%) & & \\
Yes & & \\
No & & \\
\hline
\end{tabular}

and the subsite of hypopharynx or administration of concurrent chemoradiotherapy. For patients with stage III and IV disease, the induction chemotherapy group had a relatively worse overall survival rate than the non-induction group (44\% vs. $70 \%, p=0.09)$. Multivariate analysis revealed that stage was a significant negative prognostic factor in the definitiveradiotherapy group $(p<0.01)$.

In 29 patients with stage IVA disease treated with postoperative radiotherapy, there were four locoregional recurrences (14\%) and five occurrences of distant metastasis $(17 \%)$. Salvage surgery was performed in three patients with neck lymph node metastasis and solitary liver metastasis. For patients with stage IVA disease, the 3-year locoregional control rate of postoperative radiotherapy was $83 \%$, which was significantly better than that for definitive radiotherapy at $51 \%(p=0.01$; Figure $3 \mathrm{~A})$.

During follow-up of the postoperative radiotherapy group, seven patients died of hypopharyngeal cancer and two died of intercurrent disease, including intestinal necrosis and acute heart failure. For patients with stage IVA disease, the 3-year overall survival rate for those treated with postoperative radiotherapy was $75 \%$, which was significantly better than that for those treated with definitive radiotherapy at $55 \%$

\section{$\begin{array}{lllllll}1 w & 2 w & 3 w & 4 w & 5 w & 6 w & 7 w\end{array}$

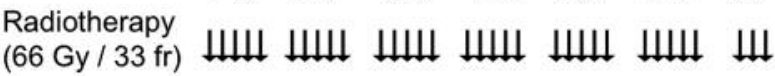

Cisplatin

$6 \mathrm{mg} / \mathrm{m}^{2}$

day $1-5 \quad 8-12 \quad 15-19 \quad 22-26$ Total 20 days

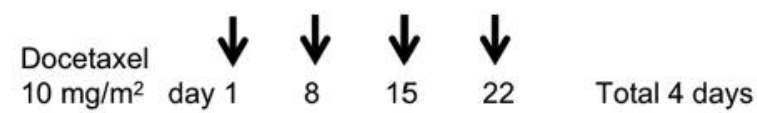

Figure 1. Regimen of concurrent chemoradiotherapy with low-dose cisplatin and docetaxel. fr: Fractions; w: week.

$(p=0.04$, Figure 3B). Multivariate analysis revealed that treatment (definitive $v s$. postoperative radiotherapy) was a significant prognostic factor for stage IVA disease $(p=0.05)$.

Of 101 patients in this study, $46(46 \%)$ had 59 synchronous or metachronous malignancies. There were 24 , 18 , and 17 patients with malignancies before, during, and after treatment for hypopharyngeal cancer, respectively. The number of patients with synchronous or metachronous malignancies were as follows: esophageal cancer, 19; gastric cancer, 10; lung cancer, 8; prostate cancer, 4; oral floor, 3; colon cancer, 3; bladder cancer, 3; skin cancer, 2; tongue cancer, 2; leukemia, 1; myelodysplastic syndromes, 1; oropharyngeal cancer, 1; pancreas cancer, 1; and thyroid cancer, 1. Eleven patients had triple malignancies, and one had a quadruple malignancy.

Acute and late adverse events are shown in Table III. With regard to acute adverse events, grade 2 to 3 radiation mucositis was common $(86 \%)$. Grade 2 to 3 dermatitis radiation was observed in 53 patients $(52 \%)$. Grade 2 hoarseness was observed in 10 patients $(10 \%)$. These acute adverse events immediately improved after the end of treatment. There were no grade 4 non-hematological events nor any treatment-related deaths during follow-up.

With regard to late adverse events, one patient (1\%) receiving definitive radiotherapy had grade 3 laryngeal stenosis and dysphagia, requiring the use of gastrostomy feeding. There were nine patients $(9 \%)$ with grade 2 xerostomia. Three patients $(3 \%)$ treated with postoperative radiotherapy had late grade 2 hypothyroidism. There were no instances of late mucositis or dermatitis radiation greater than grade 2 .

\section{Discussion}

Because most trials are performed for locally advanced head and neck cancer rather than for early-stage tumors, the treatment of stage I-II hypopharyngeal cancer is still controversial $(8,9)$. Owing to the rarity of hypopharyngeal 

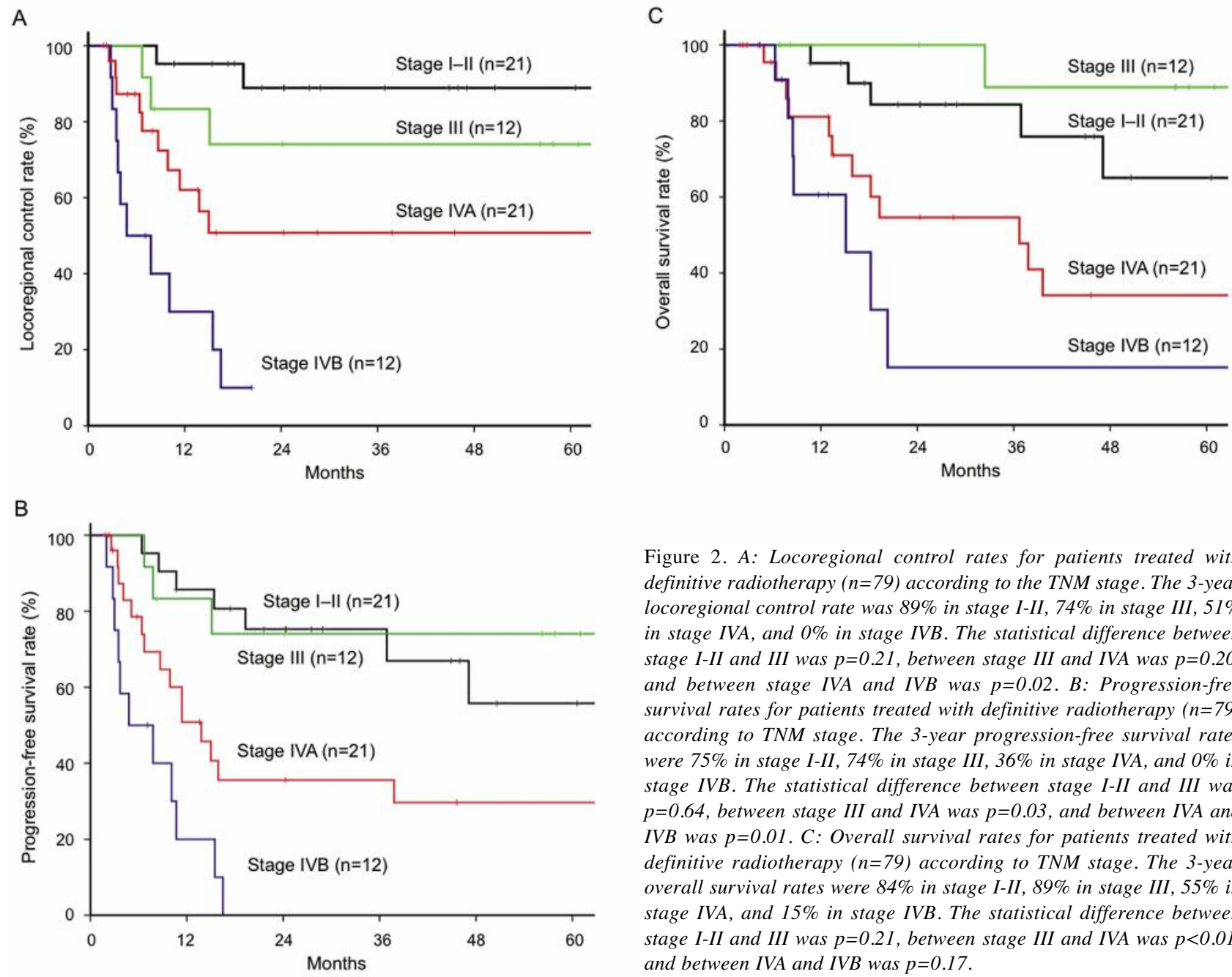

Figure 2. A: Locoregional control rates for patients treated with definitive radiotherapy $(n=79)$ according to the TNM stage. The 3-year locoregional control rate was $89 \%$ in stage I-II, 74\% in stage III, $51 \%$ in stage IVA, and $0 \%$ in stage IVB. The statistical difference between stage I-II and III was $p=0.21$, between stage III and IVA was $p=0.20$, and between stage IVA and IVB was $p=0.02$. B: Progression-free survival rates for patients treated with definitive radiotherapy $(n=79)$ according to TNM stage. The 3-year progression-free survival rates were $75 \%$ in stage I-II, 74\% in stage III, $36 \%$ in stage IVA, and $0 \%$ in stage IVB. The statistical difference between stage I-II and III was $p=0.64$, between stage III and IVA was $p=0.03$, and between IVA and IVB was $p=0.01$. C: Overall survival rates for patients treated with definitive radiotherapy $(n=79)$ according to TNM stage. The 3-year overall survival rates were $84 \%$ in stage I-II, $89 \%$ in stage III, $55 \%$ in stage IVA, and $15 \%$ in stage IVB. The statistical difference between stage I-II and III was $p=0.21$, between stage III and IVA was $p<0.01$, and between IVA and IVB was $p=0.17$.

A

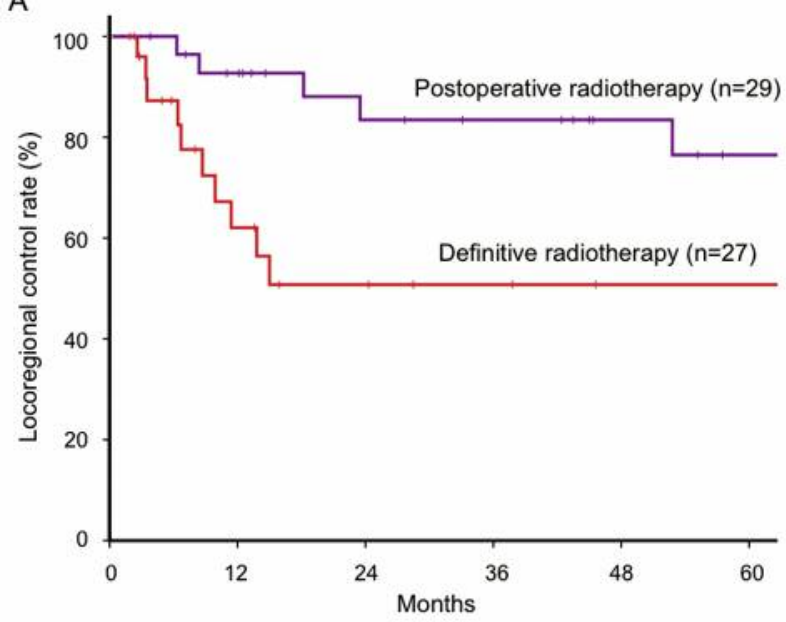

B

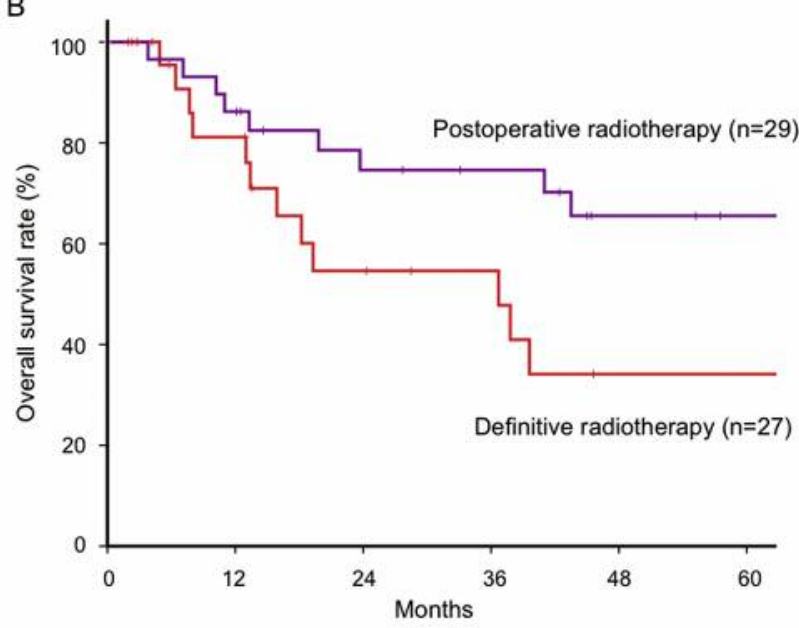

Figure 3. Locoregional control (A) and overall survival (B) for patients with stage IVA disease treated with definitive radiotherapy ( $n=27)$ and postoperative radiotherapy $(n=29)$. Postoperative radiotherapy resulted in significantly better locoregional control rate than did definitive radiotherapy $(p=0.01)$. Postoperative radiotherapy resulted in significantly better overall survival rates than did definitive radiotherapy $(p=0.04)$. 
Table II. Distribution of initial failures according to TNM stage.

\begin{tabular}{llcccccc}
\hline & \multicolumn{3}{c}{ Definitive radiotherapy } & & \multicolumn{3}{c}{ Postoperative radiotherapy } \\
\cline { 2 - 3 } Initial failure & $\begin{array}{c}\text { Stages I-II } \\
(\mathrm{n}=21)\end{array}$ & $\begin{array}{c}\text { Stage III } \\
(\mathrm{n}=12)\end{array}$ & $\begin{array}{c}\text { Stage IVA } \\
(\mathrm{n}=27)\end{array}$ & & $\begin{array}{c}\text { Stage IVB } \\
(\mathrm{n}=12)\end{array}$ & $\begin{array}{c}\text { Stage IVA } \\
(\mathrm{n}=29)\end{array}$ & $\begin{array}{c}\text { Total } \\
(\mathrm{n}=101)\end{array}$ \\
\hline Locoregional & $2(10 \%)$ & $3(25 \%)$ & $10(37 \%)$ & & $10(83 \%)$ & $4(14 \%)$ & $29(29 \%)$ \\
Distant & $1(5 \%)$ & $0(0 \%)$ & $3(11 \%)$ & & $1(8 \%)$ & $5(17 \%)$ & $10(10 \%)$ \\
Locoregional + distant & $0(0 \%)$ & $0(0 \%)$ & $0(0 \%)$ & & $0(0 \%)$ & $1(3 \%)$ & $1(1 \%)$ \\
Total & $3(14 \%)$ & $3(25 \%)$ & $13(48 \%)$ & & $11(92 \%)$ & $10(34 \%)$ & $40(40 \%)$ \\
\hline
\end{tabular}

cancer, clinical outcomes, laryngeal preservation rates, and severe adverse events have not been fully established for these patients. A few retrospective studies for early-stage hypopharyngeal cancer have reported that the overall and disease-specific survival rates achieved with definitive radiotherapy are comparable to those achieved with surgery (10-13). For example, Rabbani et al. reported good local control of $85 \%$ for T1-T2 pyriform sinus carcinoma treated with radiation alone (10). Moreover, Yoshimura et al. reported that 77 patients with stage I-II disease treated with definitive radiotherapy, including $21 \%$ with concurrent chemoradiotherapy, had a 5-year overall survival rate of $47 \%$ and a local control rate of $70 \%$ (12). In Japan, a multiinstitutional retrospective analysis of 115 patients with stage I-II hypopharyngeal cancer treated with definitive radiotherapy (50\% with concurrent chemoradiotherapy) showed that the 5-year overall survival rate was $66 \%$ and local control rate was $87 \%$ (13). In the present study, for 21 patients with stage I-II disease treated with definitive radiotherapy ( $86 \%$ with concurrent radiotherapy), the 3-year overall survival rate was $89 \%$, the locoregional control rate was $85 \%$, and laryngeal preservation was $90 \%$. Our results were comparatively better than those of previous studies, possibly due to the high rate of concurrent chemoradiotherapy. Although concurrent chemoradiotherapy has been demonstrated as an effective treatment for locally advanced head and neck cancer, its efficacy for early-stage hypopharyngeal cancer is not clear. A meta-analysis showed no benefit of chemotherapy in addition to radiotherapy in stage I-II hypopharyngeal cancer (hazard ratio=1.01) (14). Thus, further prospective study is warranted to establish the optimal combination of chemotherapy and radiotherapy for early-stage hypopharyngeal cancer.

For locally advanced hypopharyngeal cancer, prior to the 1990s, surgery was considered the only curative treatment. Several trials have been performed on laryngeal preservation in such patients without reducing survival. A randomized phase III trial comparing surgery followed by postoperative radiotherapy versus induction chemotherapy and radiotherapy was conducted by the European Organization for Research
Table III. Acute and late adverse events in all patients $(n=101)$.

\begin{tabular}{lccc}
\hline & Grade 2 & Grade 3 & Grade 4 \\
\hline $\begin{array}{l}\text { Acute adverse events, n (\%) } \\
\text { Mucositis }\end{array}$ & $70(70 \%)$ & $17(17 \%)$ & $0(0 \%)$ \\
$\quad \begin{array}{l}\text { Dermatitis radiation } \\
\text { Xerostomia }\end{array}$ & $48(49 \%)$ & $5(5 \%)$ & $0(0 \%)$ \\
$\begin{array}{l}\text { Dysgeusia } \\
\text { Hoarseness }\end{array}$ & $16(16 \%)$ & - & - \\
Late adverse events, n $(\%)$ & $10(10 \%)$ & $0(0 \%)$ & $0(0 \%)$ \\
$\quad$ Xerostomia & $9(9 \%)$ & - & - \\
$\begin{array}{l}\text { Dysgeusia } \\
\text { Pharyngeal stenosis }\end{array}$ & $2(2 \%)$ & - & - \\
Hypothyroidism & $0(0 \%)$ & $1(1 \%)$ & $0(0 \%)$ \\
\hline
\end{tabular}

- Not defined in CTCAE ver. 4.0.

and Treatment of Cancer for operable hypopharyngeal cancer patients (8). This study indicated that clinical results of the two groups were equivalent, and this was the first trial to preserve laryngeal function in patients with locally advanced hypopharyngeal cancer. Moreover, in a laryngeal cancer randomized trial, concurrent chemoradiotherapy demonstrated better locoregional control and laryngeal preservation than did induction chemotherapy followed by radiotherapy (15). Several retrospective studies also reported that concurrent chemoradiotherapy for hypopharyngeal cancer was safe and efficacious $(16,17)$. Given these prospective and retrospective studies, concurrent chemoradiotherapy has been considered an acceptable alternative to surgery. However, several chemoradiotherapy trials over the past few decades have included a variety of patients with laryngeal and hypopharyngeal cancer, making interpretation and meaningful comparisons difficult. Therefore, physicians still lack clear information on which treatments are recommended for locally advanced hypopharyngeal cancer.

In the current study, the 3-year overall survival rate for patients with stage III cancer was $89 \%$, the locoregional control rate was $74 \%$, and laryngeal preservation rate was $83 \%$, all of which were significantly better than those for 
patients with stage IVA disease. However, the clinical outcomes of stage III did not demonstrate a significant difference compared with stage I-II. Because most prior clinical studies of advanced hypopharyngeal cancer combined stages III and IV, specific results for stage III hypopharyngeal cancer have not been reported to our knowledge. Our results showed that definitive radiotherapy was effective for stage III cancer, unlike stage IV with poor prognosis. Therefore, in future prospective trials, stage III should be differentiated from stage IV, as distinct strategies may be required to treat these stages.

Whether chemoradiotherapy and radical surgery followed by postoperative radiotherapy is better for advanced hypopharyngeal cancer is controversial. However, in patients of our study with stage IVA disease, postoperative radiotherapy led to better locoregional control and overall survival rate than did definitive chemoradiotherapy. In a randomized control trial in 1997, Beauvillain et al. reported that induction chemotherapy followed by surgery gave significantly better local control and overall survival rate than did induction chemotherapy followed by radiotherapy (5-year local control rate, 63\% vs. 39\%; 5-year overall survival rate, $37 \%$ vs. $19 \%$, respectively) (18). Furthermore, a study of the National Cancer Database showed that total laryngectomy led to a significantly better overall survival rate than did chemoradiotherapy for laryngeal preservation in patients with T4a laryngeal cancer (19). Harris et al. retrospectively compared primary surgery followed by radiotherapy to definitive radiotherapy in locally advanced hypopharyngeal cancer, including $82 \%$ with stage IV (20). Primary surgery followed by radiotherapy tended to improve the overall survival rate compared with that of definitive radiotherapy $(p=0.06)$. Given these findings, surgery followed by radiotherapy may provide superior results than definitive radiotherapy in stage IVA hypopharyngeal cancer. However, careful interpretation is required due to selection bias. For example, $60 \%$ of stage IVA cancer cases treated with definitive chemoradiotherapy in the current study were diagnosed as being inoperable. Furthermore, patients with neck swelling and a poor prognosis were treated more often with definitive radiotherapy than with surgery followed by postoperative radiotherapy. Therefore, operable and mildly symptomatic patients tended to receive surgery. Accordingly, a prospective study to compare definitive chemoradiotherapy and postoperative radiotherapy is required for appropriate patient selection.

Because most trials excluded highly advanced hypopharyngeal cancer, few studies have evaluated stage IVB hypopharyngeal cancer. In the current study, stage IVB was medically inoperable because of tumor invasion to prevertebral fascia, encasement of the carotid artery, or metastasis in a lymph node $>6 \mathrm{~cm}$ in diameter. Although chemoradiotherapy is considered radical therapy, the treatment results at our facility were not acceptable: the 3year locoregional control rate was $0 \%$, and overall survival rate was $15 \%$, both of which were significantly worse outcomes than those for patients with stage IVA disease. Only one patient surviving for a longer time underwent salvage surgery for residual tumor after radiotherapy. Aggressive treatment strategies, such as combined therapy chemoradiotherapy and surgery, intensive chemotherapy regimens, or radiation dose escalation, were required to overcome this intractable disease.

Concurrent chemoradiotherapy (70 Gy in 35 fractions) with high-dose cisplatin is considered a standard regimen for head and neck cancer (4). However, intensive chemoradio-therapy induced late severe adverse effects, such as requiring a feeding tube or causing aspiration pneumonia (21). In our study, concurrent chemoradiotherapy with a low-dose cisplatin and docetaxel regimen was mainly used (74\%). Previously, we reported the feasibility and efficacy of this regimen for head and neck cancer, with 2-year local control of $90 \%$ without severe toxicity (6). The current study showed that a low-dose cisplatin and docetaxel regimen frequently resulted in acute mucositis and dermatitis, but not in late adverse events. Inohara et al. performed a phase II study of low-dose cisplatin and docetaxel of six courses with 66 Gy for 117 patients with head and neck squamous cell carcinomas (47\% with hypopharyngeal cancer), resulting in a complete response rate of $71 \%$ and a 4 year overall survival rate of $68 \%$ (22). Although acute mucositis was common, feeding tube dependence was $8 \%$ and tracheostomy was $1 \%$ in surviving patients. Salvage surgery is feasible, and there were no late tissue radiation sequelae to exclude salvage surgery. Given these findings, the addition of docetaxel to cisplatin can improve local control without severe late adverse events, which is considered to be an effective chemoradiotherapy regimen.

As described above, induction chemotherapy followed by radiotherapy alone was developed for laryngeal preservation in a trial on advanced hypopharyngeal cancer (9). Induction chemotherapy is expected to reduce metastasis and reduce tumor burden before radiotherapy $(23,24)$. However, in the era of concurrent chemoradiotherapy, additional induction chemotherapy has been controversial for hypopharyngeal cancer. For head and neck cancer, there have been several randomized trials comparing induction chemotherapy followed by concurrent chemoradiotherapy and primary concurrent chemoradiotherapy (25-27). However, additional induction treatment did not improve overall survival, and severe toxicity was frequently observed due to cumulative chemotherapy (25). A meta-analysis of radiotherapy and chemotherapy in head and neck cancer showed that there was no clear evidence of a benefit for induction chemotherapy (28). Furthermore, in a retrospective study, Nakahara et al. reported that induction chemotherapy had significantly worse outcomes than those of non-induction chemotherapy in patients with hypopharyngeal cancer (29). For stage III-IV disease in the current study, the induction chemotherapy group had worse locoregional control and overall 
survival rates than did the non-induction group. Biologically, induction chemotherapy could delay the need for strong locoregional treatment (e.g. concurrent chemoradiotherapy), which selects surviving cells resistant to radiotherapy. Thus, unless the efficacy of induction chemotherapy is demonstrated for advanced-stage hypopharyngeal cancer, concurrent chemoradiotherapy can remain the standard of care (30).

Forty-six percent of patients with hypopharyngeal cancer in the present study had synchronous or metachronous malignancies, and $4 \%$ died from these tumors. In hypopharyngeal cancer, secondary primary tumors of the aerodigestive tract are often observed due to consumption of cigarettes and alcohol. A Japanese multi-institutional retrospective analysis for stage I-II hypopharyngeal cancer reported that $56.5 \%$ of patients had synchronous or metachronous malignancies, and the authors concluded that esophageal cancer had a major impact on overall survival (13). In addition, Yoshimura et al. reported that patients with advanced-stage synchronous or metachronous malignancies had significantly worse survival rates than those with earlystage malignancies (12). Therefore, in order to avoid death from metachronous malignancies after treatment of hypopharyngeal cancer, careful follow-up for detecting earlystage malignancies is mandatory.

Our study has a few limitations, such as that it was retrospective and included several types of chemotherapy and radiation treatment techniques over more than a decade. However, owing to the rarity of hypopharyngeal cancer, clinical outcomes of definitive and postoperative radiotherapy have not fully been evaluated. Therefore, it is important to accumulate patients and evaluate results. A multi-institutional retrospective study is warranted to evaluate the efficacy and safety of radiotherapy and to support developing future clinical trials for early and advanced hypopharyngeal cancer.

\section{Conclusion}

Definitive radiotherapy had the highest efficacy regarding locoregional control and overall survival rates with a high laryngeal preservation rate in stage I-II disease. Among those with advanced disease treated by definitive radiotherapy, those with stage III disease had better clinical outcomes than those with stage IVA-B. Even if local recurrence developed after radiotherapy, salvage surgery was safely performed, which improved the survival rate without postoperative complications. Concurrent chemoradiotherapy with low-dose cisplatin and docetaxel frequently developed acute mucositis and dermatitis, but not severe late adverse events, indicating that treatments were safe. Although definitive radiotherapy was an effective initial treatment option for stage I-III hypopharyngeal cancer, the clinical outcome of stage IVA-B was unsatisfactory. For stage IVA, definitive radiotherapy resulted in a significantly worse prognosis than did postoperative radiotherapy. The results regarding stage IVB were poor, and further treatment methods are required to improve survival. Especially in advanced-stage disease, this study showed that stage III, IVA and IVB, which are considered equally as locally advanced hypopharyngeal cancer in most clinical trials, had distinct clinical aspects and outcomes; accordingly, they should be categorized by specific stages and treated with optimized therapy for each stage.

\section{Acknowledgements}

The Authors wish to thank Editage (www.editage.jp) for English language editing.

This work was supported by JSPS KAKENHI Grant Number 26461878.

\section{References}

1 Takes RP, Strojan P, Silver CE, Bradley PJ, Haigentz M Jr., Wolf GT, Shaha AR, Hartl DM, Olofsson J, Langendijk JA, Rinaldo A, Ferlito A and International Head and Neck Scientific Group: Current trends in initial management of hypopharyngeal cancer: the declining use of open surgery. Head Neck 34: 270-281, 2012.

2 Godballe $\mathrm{C}$, Jørgensen $\mathrm{K}$, Hansen $\mathrm{O}$ and Bastholt L: Hypopharyngeal cancer: results of treatment based on radiation therapy and salvage surgery. Laryngoscope 112: 834-838, 2002.

3 Adelstein DJ, Li Y, Adams GL, Wagner H Jr., Kish JA, Ensley JF, Schuller DE and Forastiere AA: An intergroup phase III comparison of standard radiation therapy and two schedules of concurrent chemoradiotherapy in patients with unresectable squamous cell head and neck cancer. J Clin Oncol 21: 92-98, 2003.

4 Ang KK, Harris J, Garden AS, Trotti A, Jones CU, Carrascosa L, Cheng JD, Spencer SS, Forastiere A and Weber RS: Concomitant boost radiation plus concurrent cisplatin for advanced head and neck carcinomas: Radiation Therapy Oncology Group phase II trial 99-14. J Clin Oncol 23: 3008-3015, 2005.

5 Pignon JP, Bourhis J, Domenge C and Designé L: Chemotherapy added to locoregional treatment for head and neck squamous-cell carcinoma: three meta-analyses of updated individual data. MACH-NC Collaborative Group. Meta-analysis of chemotherapy on head and neck cancer. Lancet 355: 949-955, 2000.

6 Kitamoto Y, Akimoto T, Ishikawa H, Nonaka T, Katoh H, Nakano T, Ninomiya H, Chikamatsu K and Furuya N: Acute toxicity and preliminary clinical outcomes of concurrent radiation therapy and weekly docetaxel and daily cisplatin for head and neck cancer. Jpn J Clin Oncol 35: 639-644, 2005.

7. Common Terminology Criteria for Adverse Events version 4.0 http://ctep.cancer.gov/protocolDevelopment/electronic_applicatio ns/ctc.htm\#ctc_40

8 Lefebvre JL, Chevalier D, Luboinski B, Kirkpatrick A, Collette L and Sahmoud T: Larynx preservation in pyriform sinus cancer: preliminary results of a European Organization for Research and Treatment of Cancer phase III trial. EORTC Head and Neck Cancer Cooperative Group. J Natl Cancer Inst 88: 890-899, 1996.

9 Lefebvre JL, Rolland F, Tesselaar M, Bardet E, Leemans CR, Geoffrois L, Hupperets P, Barzan L, de Raucourt D, Chevalier D, Licitra L, Lunghi F, Stupp R, Lacombe D, Bogaerts J, Horiot JC, Bernier J, Vermorken JB and EORTC Head and Neck Cancer Cooperative Group; EORTC Radiation Oncology Group: 
Phase 3 randomized trial on larynx preservation comparing sequential $v s$. alternating chemotherapy and radiotherapy. J Natl Cancer Inst 101: 142-152, 2009.

10 Rabbani A, Amdur RJ, Mancuso AA, Werning JW, Kirwan J, Morris CG and Mendenhall WM: Definitive radiotherapy for T1T2 squamous cell carcinoma of pyriform sinus. Int J Radiat Oncol Biol Phys 72: 351-355, 2008.

11 Garden AS, Morrison WH, Clayman GL, Ang KK and Peters LJ: Early squamous cell carcinoma of the hypopharynx: outcomes of treatment with radiation alone to the primary disease. Head Neck 18: 317-322, 1996.

12 Yoshimura R, Kagami Y, Ito Y, Asai M, Mayahara H, Sumi M and Itami $\mathrm{J}$ : Outcomes in patients with early-stage hypopharyngeal cancer treated with radiotherapy. Int J Radiat Oncol Biol Phys 77: 1017-1023, 2010.

13 Nakamura K, Shioyama Y, Kawashima M, Saito Y, Nakamura N, Nakata K, Hareyama M, Takada T, Karasawa K, Watanabe T, Yorozu A, Tachibana H, Suzuki G, Hayabuchi N, Toba T and Yamada S: Multi-institutional analysis of early squamous cell carcinoma of the hypopharynx treated with radical radiotherapy. Int J Radiat Oncol Biol Phys 65: 1045-1050, 2006.

14 Blanchard P, Baujat B, Holostenco V, Bourredjem A, Baey C, Bourhis J, Pignon JP and MACH-CH Collaborative group: Meta-analysis of chemotherapy in head and neck cancer (MACH-NC): a comprehensive analysis by tumour site. Radiother Oncol 100: 33-40, 2011.

15 Forastiere AA, Goepfert H, Maor M, Pajak TF, Weber R, Morrison W, Glisson B, Trotti A, Ridge JA, Chao C, Peters G, Lee DJ, Leaf A, Ensley $\mathrm{J}$ and Cooper J: Concurrent chemotherapy and radiotherapy for organ preservation in advanced laryngeal cancer. N Engl J Med 349: 2091-2098, 2003.

16 Samant S, Kumar P, Wan J, Hanchett C, Vieira F, Murry T, Wong FS and Robbins KT: Concomitant radiation therapy and targeted cisplatin chemotherapy for the treatment of advanced pyriform sinus carcinoma: disease control and preservation of organ function. Head Neck 21: 595-601, 1999.

17 Tai SK, Yang MH, Wang LW, Tsai TL, Chu PY, Wang YF, Huang JL and Chang SY: Chemoradiotherapy laryngeal preservation for advanced hypopharyngeal cancer. Jpn J Clin Oncol 38: 521-527, 2008.

18 Beauvillain C, Mahé M, Bourdin S, Peuvrel P, Bergerot P, Rivière A, Vignoud J, Deraucourt D and Wesoluch M: Final results of a randomized trial comparing chemotherapy plus radiotherapy with chemotherapy plus surgery plus radiotherapy in locally advanced resectable hypopharyngeal carcinomas. Laryngoscope 107: 648-653, 1997.

19 Grover S, Swisher-McClure S, Mitra N, Li J, Cohen RB, Ahn PH, Lukens JN, Chalian AA, Weinstein GS, O'Malley BW Jr. and Lin A: Total laryngectomy versus larynx preservation for T4a larynx cancer: patterns of care and survival outcomes. Int $\mathbf{J}$ Radiat Oncol Biol Phys 92: 594-601, 2015.

20 Harris BN, Biron VL, Donald P, Farwell DG, Luu QC, Bewley AF, Chen AM and Daly ME: Primary surgery vs. chemoradiation treatment of advanced-stage hypopharyngeal squamous cell carcinoma. JAMA Otolaryngol Head Neck Surg 141: 636-640, 2015.

21 Machtay M, Moughan J, Trotti A, Garden AS, Weber RS, Cooper JS, Forastiere A and Ang KK: Factors associated with severe late toxicity after concurrent chemoradiation for locally advanced head and neck cancer: an RTOG analysis. J Clin Oncol 26: 3582-3589, 2008.
22 Inohara H, Takenaka Y, Yoshii T, Nakahara S, Yamamoto Y, Tomiyama Y, Seo Y, Isohashi F, Suzuki O, Yoshioka Y, Sumida I and Ogawa K: Phase 2 study of docetaxel, cisplatin, and concurrent radiation for technically resectable stage III-IV squamous cell carcinoma of the head and neck. Int J Radiat Oncol Biol Phys 91: 934-941, 2015.

23 Posner MR, Hershock DM, Blajman CR, Mickiewicz E, Winquist E, Gorbounova V, Tjulandin S, Shin DM, Cullen K, Ervin TJ, Murphy BA, Raez LE, Cohen RB, Spaulding M, Tishler RB, Roth B, Viroglio Rdel C, Venkatesan V, Romanov I, Agarwala S, Harter KW, Dugan M, Cmelak A, Markoe AM, Read PW, Steinbrenner L, Colevas AD, Norris CM Jr., Haddad RI and TAX 324 Study Group: Cisplatin and fluorouracil alone or with docetaxel in head and neck cancer. N Engl J Med 357: 1705-1715, 2007.

24 Vermorken JB, Remenar E, van Herpen C, Gorlia T, Mesia R, Degardin M, Stewart JS, Jelic S, Betka J, Preiss JH, van den Weyngaert D, Awada A, Cupissol D, Kienzer HR, Rey A, Desaunois I, Bernier J, Lefebvre JL and EORTC 24971/TAX 323 Study Group: Cisplatin, fluorouracil, and docetaxel in unresectable head and neck cancer. N Engl J Med 357: 16951704, 2007.

25 Haddad R, O'Neill A, Rabinowits G, Tishler R, Khuri F, Adkins D, Clark J, Sarlis N, Lorch J, Beitler JJ, Limaye S, Riley S and Posner M: Induction chemotherapy followed by concurrent chemoradiotherapy (sequential chemoradiotherapy) versus concurrent chemoradiotherapy alone in locally advanced head and neck cancer (PARADIGM): a randomised phase 3 trial. Lancet Oncol 14: 257-264, 2013.

26 Cohen EE, Karrison TG, Kocherginsky M, Mueller J, Egan R, Huang CH, Brockstein BE, Agulnik MB, Mittal BB, Yunus F, Samant S, Raez LE, Mehra R, Kumar P, Ondrey F, Marchand P, Braegas B, Seiwert TY, Villaflor VM, Haraf DJ and Vokes EE: Phase III randomized trial of induction chemotherapy in patients with N2 or N3 locally advanced head and neck cancer. J Clin Oncol 32: 2735-2743, 2014.

27 Hitt R, Grau JJ, López-Pousa A, García-Girón C, Irigoyen A, Sastre J, Martínez-Trufero J, Brandariz Castelo JA, Verger E, CruzHernández JJ and Spanish Head and Neck Cancer Cooperative Group (TTCC): A randomized phase III trial comparing induction chemotherapy followed by chemoradiotherapy versus chemoradiotherapy alone as treatment of unresectable head and neck cancer. Ann Oncol 25: 216-225, 2014.

28 Pignon JP, le Maître A, Maillard E, Bourhis J and MACH-NC Collaborative Group: Meta-analysis of chemotherapy in head and neck cancer (MACH-NC): an update on 93 randomised trials and 17,346 patients. Radiother Oncol 92: 4-14, 2009.

29 Nakahara R, Kodaira T, Furutani K, Tachibana H, Tomita N, Inokuchi H, Mizoguchi N, Goto Y, Ito Y and Naganawa S: Treatment outcomes of definitive chemoradiotherapy for patients with hypopharyngeal cancer. J Radiat Res 53: 906-915, 2012.

30 Gyawali B, Shimokata T, Honda K and Ando Y: Chemotherapy in locally advanced head and neck squamous cell carcinoma. Cancer Treat Rev 44: 10-16, 2016. 